PROCEEDINGS OF THE

AMERICAN MATHEMATICAL SOCIETY

Volume 135, Number 9, September 2007, Pages 2743-2746

S 0002-9939(07)08816-8

Article electronically published on May 8, 2007

\title{
ON $U(p)$-CONGRUENCES
}

\author{
P. GUERZHOY
}

(Communicated by Ken Ono)

\begin{abstract}
The phenomenon of $U(p)$-congruences was recently studied by Ahlgren and Ono (2005) and by Elkies, Ono and Yang (2005). We provide a necessary and sufficient condition which improves their general results.
\end{abstract}

Let

$$
j(\tau)=q^{-1}+744+196884 q+21493760 q^{2}+\cdots \in \frac{1}{q} \mathbb{Z}[[q]]
$$

be the usual elliptic modular function on $S L_{2}(\mathbb{Z})\left(q:=e^{2 \pi i \tau}\right.$ throughout). Recall that a modular form (on $S L_{2}(\mathbb{Z})$ ) is called weakly holomorphic if its only pole is at infinity. Every weakly holomorphic modular form of weight zero $f$ on $S L_{2}(\mathbb{Z})$ is a polynomial $F$ in $j$ and has a $q$-expansion of the form

$$
f=F(j)=\sum_{n \gg-\infty} a(n) q^{n} .
$$

For a rational prime $p$ the action of the $U=U(p)$-operator on formal power series in the variable $q$ is defined by $F \mapsto F \mid U$, where $F=\sum a(n) q^{n}$ and $F \mid U=\sum a(p n) q^{n}$. Denote by $\bar{j} \in \mathbb{F}_{p}[[q]]$ the modulo $p$ coefficient-wise reduction of $j$. Recently Elkies, Ono and Yang in [2] considered the following question. For a monic polynomial $F(x) \in \mathbb{F}_{p}[x]$, under which conditions does there exist a polynomial $G(x) \in \mathbb{F}_{p}[x]$ such that

$$
F(\bar{j}) \mid U=G(\bar{j})
$$

as power series in $\mathbb{F}_{p}[[q]]$ ? Note that $\operatorname{deg}(G) \leq \operatorname{deg}(F) / p$ since $j=q^{-1}+O(1)$. A special case of this question with $\operatorname{deg}(F)<p$ was considered in a recent paper by Ahlgren and Ono [1]. In this case $\operatorname{deg}(G)=0$ or $-\infty$, and the question specializes as follows. For a polynomial $F(x) \in \mathbb{F}_{p}[x]$ of degree $<p$, under which conditions does the congruence

$$
F(j) \mid U \equiv a(0) \bmod p
$$

hold? Ono in 4 calls (2) a $U(p)$-congruence. We adopt this terminology and generalize it to (1).

In this note we prove a simple necessary and sufficient condition for the $U(p)$ congruence to hold.

Note that general $U(p)$-congruences are not the primary focus of [1] and [2] . These projects are devoted to the interplay between singular moduli and class

Received by the editors May 2, 2006 and, in revised form, June 6, 2006.

2000 Mathematics Subject Classification. Primary 11F33.

The research of this author was supported by NSF grant DMS-0501225.

(C)2007 American Mathematical Society Reverts to public domain 28 years from publication 
polynomials. In particular, general criteria for an arbitrary (monic) polynomial $F$ to satisfy a $U(p)$-congruence were obtained in loc. cit. and were applied to the case when $F$ is a Hilbert class polynomial.

The author wants to thank Ken Ono for a valuable suggestion to prove a result in the generality corresponding to [2], whereas the initial version of this paper was bounded by the framework of [1].

Following [1] and 2], consider an arbitrary rational prime $p$ and introduce the polynomial $S_{p}(x) \in \mathbb{F}_{p}[x]$ of degree $\left\lfloor\frac{p}{12}\right\rfloor$, which is a slight modification of the supersingular polynomial at $p$. More specifically, let $S_{p}(x)=1$ if $p \leq 11$, and for $p>11$ let

$$
S_{p}(x)=\prod_{\substack{E / \mathbb{F}_{p} \\ j(E) \notin\{e r s i n g u l a r}}(x-j(E)) \in \mathbb{F}_{p}[x],
$$

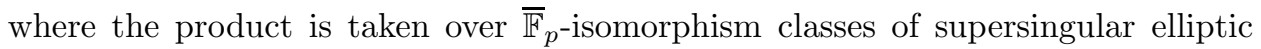
curves $E$.

The general results pertaining to $U(p)$-congruences, proved in [1] and 2], may be summarized as follows.

\section{Theorem 0.}

(i) (11, Theorem 2], [2, Theorem 2.3]) If $S_{p}(x)^{2} \mid F(x)$, then $F$ satisfies a $U(p)$ congruence.

(ii) ([1, Corollary 5], [2]) If $F$ satisfies a $U(p)$-congruence, then $p \leq 12 \operatorname{deg}(F)+1$.

It is mentioned in [1, Theorem 2, Remark 1] that the sufficient double divisibility condition (i) is not necessary; the necessary condition (ii) is not sufficient. We present a necessary and sufficient condition in this paper.

Theorem 1. Assume that $p>3$. A monic polynomial $F(x) \in \mathbb{F}_{p}[x]$ satisfies a $U(p)$-congruence if and only if its derivative $F^{\prime}(x)$ is divisible by $S_{p}(x)$ in $\mathbb{F}_{p}[x]$.

Remarks.

(1) Our Theorem 1 immediately implies Theorem 0. Indeed, if $S_{p}(x)^{2} \mid F(x)$, then $S_{p} \mid F^{\prime}(x)$ (but not conversely), and, by Theorem $1, F$ satisfies a $U(p)$-congruence, which proves (i). Conversely, if $F$ satisfies a $U(p)$-congruence, then, by Theorem 1, $S_{p} \mid F^{\prime}(x)$, and therefore $p \leq 12 \operatorname{deg}(F)+1$ since $\operatorname{deg} S_{p}=\left\lfloor\frac{p}{12}\right\rfloor$, which proves (ii).

(2) One can slightly generalize the notion of $U(p)$-congruences (2) allowing repeated application of the $U$-operator. This generalization, however, does not bring anything new as soon as the degree of $F$ is smaller than $p$. Using a result of Serre [5. Théorème 6 ii] (and our proof of Theorem 1) one proves that if the congruence

$$
F(j) \mid U^{m} \equiv a(0) \bmod p
$$

holds with some $m \geq 1$, then it holds for any $m \geq 1$.

(3) If $p \leq 11$ further divisibilities appear. For an arbitrary polynomial $F(x) \in$ $\mathbb{Z}[x]$ and any positive integer $\alpha$ the results of Serre [5, Lemme 3] for $p<11$, [5. Théorème 8, Exemple 1] and [6, Théorème 5.4] allow one to prove that the congruence

holds if $m$ is big enough.

$$
F(j) \mid U^{m} \equiv a(0) \bmod p^{\alpha}
$$

Our proof of Theorem 1 is based on an idea of Serre [ 6 , 6.16b], where a special case of a similar argument $(F(x)=x$ in loc. cit.) was left as an exercise to the reader. 
Proof. We use the standard (Ramanujan) notations for the Eisenstein series of weights 4 and 6 and for the normalized cusp form of weight 12:

$$
Q=1+240 \sum_{n \geq 1} \sigma_{3}(n) q^{n}, \quad R=1-504 \sum_{n \geq 1} \sigma_{5}(n) q^{n}, \quad \Delta=\frac{1}{1728}\left(Q^{3}-R^{2}\right) .
$$

We denote by $\bar{Q}, \bar{R}$ and $\bar{\Delta} \in \mathbb{F}_{p}[[q]]$ the coefficientwise modulo $p$ reductions of the above series. The operator $D$ (sometimes denoted by $\theta$ ) acts on formal $q$-series and takes $\sum a(n) q^{n}$ to $\sum n a(n) q^{n}$.

We begin with the identity $(F \mid U)^{p}=F-D^{p-1} F$ of power series over $\mathbb{F}_{p}$, which implies the identity

$$
\bar{\Delta}^{b p}(F \mid U)^{p}=\bar{\Delta}^{b p} F-\bar{\Delta}^{b p} D^{p-1} F
$$

for any positive integer $b$. Recall that a modulo $p$ modular form is a power series in $\mathbb{F}_{p}[[q]]$ which coincides with a modulo $p$ reduction of the $q$-expansion of a holomorphic modular form on $S L(2, \mathbb{Z})$ with rational $p$-integral $q$-expansion coefficients. We denote the space of modulo $p$ modular forms by $M$. For $f \in M$ we denote by $w(f)$ its filtration. The definition and a detailed exposition of the theory of filtration are contained in [5, 6, 7].

If $b$ is big enough (i.e. $b>(\operatorname{deg} F) / p$ ) all three terms in (3) belong to $M$. Indeed, evidently $\bar{\Delta}^{b p} F \in M$. Also $\bar{\Delta}^{b p}(F \mid U)^{p} \in M$ by [6, Théorème 5.4]. Then (3) implies $\bar{\Delta}^{b p} D^{p-1} F \in M$. Thus the filtrations of these terms are well-defined. We are particularly interested in $w\left(\bar{\Delta}^{b}(F \mid U)\right)$, and we are going to estimate the filtrations of the three terms in (3) in order to conclude that

$$
w\left(\bar{\Delta}^{b}(F \mid U)\right) \quad \begin{cases}=12 b+p-1 & \text { if } S_{p}(x) \nmid F^{\prime}(x) \text { in } \mathbb{F}_{p}[x], \\ <12 b+p-1 & \text { if } S_{p}(x) \mid F^{\prime}(x) \text { in } \mathbb{F}_{p}[x] .\end{cases}
$$

We have

$$
w\left(\bar{\Delta}^{b p} F\right) \leq 12 b p .
$$

It follows from [5, Lemme 1(b)] that

$$
w\left(\bar{\Delta}^{b p}(F \mid U)^{p}\right)=w\left(\left(\bar{\Delta}^{b}(F \mid U)\right)^{p}\right)=p w\left(\bar{\Delta}^{b}(F \mid U)\right) .
$$

We now want to calculate the filtration $w\left(\bar{\Delta}^{b p} D^{p-1} F\right)$. The identity $D(j)=$ $-Q^{2} R / \Delta$, the chain rule for $a=0$, the product rule for $a>0$ ( $D$ is a differential operator), and an induction argument in $a$ imply that $\Delta^{b p} D^{a+1} F \equiv$ $-D^{a}\left(F^{\prime} Q^{2} R \Delta^{b p-1}\right) \bmod p$ for $a \geq 0$. Pick $a=p-2$ and pass to the modulo $p$ reductions to obtain

$$
w\left(\bar{\Delta}^{b p} D^{p-1} F\right)=w\left(D^{p-2}\left(F^{\prime} \bar{Q}^{2} \bar{R} \bar{\Delta}^{b p-1}\right)\right) .
$$

We use the equality

$$
w\left(\bar{\Delta}^{m} f\right)=12 m+w(f) \text { for any positive integer } m \text { and } f \in M,
$$

which follows from [3, §10.7, Lemma (i)] (cf. the proof of [7, Lemma 5]) and [7, Lemma 5(i)] to conclude that

$$
w\left(F^{\prime} \bar{Q}^{2} \bar{R} \bar{\Delta}^{b p-1}\right) \begin{cases}=12 b p+2 & \text { if } S_{p}(x) \nmid F^{\prime}(x) \text { in } \mathbb{F}_{p}[x], \\ <12 b p+2 & \text { if } S_{p}(x) \mid F^{\prime}(x) \text { in } \mathbb{F}_{p}[x] .\end{cases}
$$


Notice that $12 b p+2 \equiv 2 \bmod p$ and apply [7, Lemma 5(ii)] combined with the identity (77) to conclude that

$$
w\left(\bar{\Delta}^{b p} D^{p-1} F\right) \begin{cases}=p^{2}+(12 b-1) p & \text { if } S_{p}(x) \nmid F^{\prime}(x) \text { in } \mathbb{F}_{p}[x], \\ <p^{2}+(12 b-1) p & \text { if } S_{p}(x) \mid F^{\prime}(x) \text { in } \mathbb{F}_{p}[x] .\end{cases}
$$

Filtration satisfies the ultrametric inequality $w(f+g) \leq \sup (w(f), w(g))$ for $f, g \in M$, which implies that $w(f+g)=\max (w(f), w(g))$ provided that $w(f) \neq$ $w(g)$. We apply this observation to (3), taking into account (5), (6), (8), and the evident inequality $p^{2}+(12 b-1) p>12 p b$, and obtain

$$
p w\left(\bar{\Delta}^{b}(F \mid U)\right) \quad \begin{cases}=p^{2}+(12 b-1) p & \text { if } S_{p}(x) \nmid F^{\prime}(x) \text { in } \mathbb{F}_{p}[x], \\ <p^{2}+(12 b-1) p & \text { if } S_{p}(x) \mid F^{\prime}(x) \text { in } \mathbb{F}_{p}[x],\end{cases}
$$

which implies (4).

We now derive the statement of Theorem 1 from (4). Assume that $S_{p}(x) \mid F^{\prime}(x)$ in $\mathbb{F}_{p}[x]$. It follows from [6, Théorème 5.4], that $\Delta^{b}(F \mid U)$ is a modulo $p$ reduction of a $p$-adic modular form of weight $12 b$. Therefore by [5, Théorème 1] its filtration is $w\left(\Delta^{b}(F \mid U)\right)=w\left(\bar{\Delta}^{b}(F \mid U)\right) \equiv 12 b \bmod p-1$. This observation together with the inequality in (4) implies that $w\left(\bar{\Delta}^{b}(F \mid U)\right) \leq 12 b$. Thus there exists a modular form $f$ on $S L_{2}(\mathbb{Z})$ of weight $12 b$ such that $f \equiv \Delta^{b}(F \mid U) \bmod p$. It follows that $(F \mid U) \equiv f / \Delta^{b} \bmod p$. However, $f / \Delta^{b}$ is a modular form on $S L_{2}(\mathbb{Z})$ of weight zero with its only pole at infinity, which must be a polynomial in $j$. This proves that $F(x)$ satisfies a $U(p)$-congruence if $S_{p}(x) \mid F^{\prime}(x)$ in $\mathbb{F}_{p}[x]$.

Conversely, if the congruence (11) holds, then

$$
w\left(\Delta^{b}(F \mid U)\right)=w\left(\Delta^{b} G\right) \leq 12 b,
$$

and it follows from (4) that $S_{p}(x) \mid F^{\prime}(x)$ in $\mathbb{F}_{p}[x]$.

\section{REFERENCES}

[1] Ahlgren, Scott; Ono, Ken, Arithmetic of singular moduli and class polynomials, Compos. Math. 141 (2005), no. 2, 293-312. MR2134268 (2006a:11058)

[2] Elkies, Noam; Ono, Ken; Yang, Tonghai, Reduction of CM elliptic curves and modular function congruences, Int. Math. Res. Not. (2005), no. 44, 2695-2707. MR2181309 (2006k:11076)

[3] Lang, Serge, Introduction to modular forms, Grundlehren der mathematischen Wissenschaften, No. 222, Springer-Verlag, Berlin-New York, 1976. MR0429740 (55:2751)

[4] Ono, Ken, The Web of Modularity: Arithmetic of the Coefficients of Modular Forms and $q$ series, CBMS Reg. Conf. Ser. Math., 102, Amer. Math. Soc., Providence, RI, 2004. MR2020489 (2005c:11053)

[5] Serre, Jean-Pierre, Formes modulaires et fonctions zêta $p$-adiques, Modular functions of one variable, III, Proc. Internat. Summer School, Univ. Antwerp, 1972, pp. 191-268, Lecture Notes in Math., Vol. 350, Springer, Berlin, 1973. MR0404145(53:7949a)

[6] Serre, Jean-Pierre, Divisibilité de certaines fonctions arithmétiques, Enseignement Math. (2) 22 (1976), no. 3-4, 227-260. MR0434996 (55:7958)

[7] Swinnerton-Dyer, H. P. F., On l-adic representations and congruences for coefficients of modular forms, Modular functions of one variable, III (Proc. Internat. Summer School, Univ. Antwerp, 1972), pp. 1-55, Lecture Notes in Math., Vol. 350, Springer, Berlin, 1973. MR0406931(53:10717a)

Department of Mathematics, University of Hawail at Manoa, 2565 McCarthy Mall, Honolulu, Hawail 96822-2273

E-mail address: p.guerzhoy@gmail.com 